Eine Klammer zum Festhalten der Röhrchen bei Schmelzpunktsbestimmungen hat Alfred Köllike ${ }^{*}$ ) construirt. Dieselbe ist in Fig. 37 abgebildet. Sie besteht aus einer federnden Platte von Platinblech, die sich mit ihren verbreiterten, gebogenen Enden ${ }^{-}$um das Thermometer legt. An dieses Blech ist ein aus zwei Armen a und b bestehender Ring von Platindraht angenietet, der das Thermometer umfasst und mit dem federnỏen Platinblech zusantmen die ganze Vorrichtung daran festhält. Der Arm b ist so gebogen, dass er zwei Oesen bildet, durch welche man das zur. Schmelzpunktsbestimmung dienende Röhrchen hindurch schiebt, und welche dasselbe dann genau dem Thermometer parallel festhalten.

Der Gebrauch von weissem Licht bei dem Polaristrobometer von Wild ${ }^{* *}$ ) ist von diesem Autor dadurch ermöglicht worden, dass er das Licht durch eine achromatische Linse in den Apparat eintreten - lässt und zwischen die Röhre mit Zuckerlösung (resp. einer anderen zu untersuchenden Substanz) und das zweite Nicol'sche Prisma ein stark dispergirendes (5 faches A $\mathrm{mici}$ 'sches) Prisma einfügt. Die aus der Zuckerlösung austretenden Strahlen werden dadurch dispergirt, und man kann nun den an einem Charnier angebrachten Analysator sammt Beobachtungsfernrohr anf jeden beliebigen Theil des Spectrums einstellen und so für diese bestimmte Lichtart die durch die zu untersuchende Substanz hervorgebrachte Drehung beobachten. Zur Einstellung der gewünschten Lichtart entfernt man am besten ein Nicol'sches Prisma

*) Inauguraldissertation: Ueber die Einwirkung von Triphenylbrommethan auf Natriumacetessigester. Vorgelegt der philosoph. Facultät der Universität Freiburg i. B. Würzburg 1883 p. 24. Vom Verfasser eingesandt.

**) Bull. de l'Acad. imp. de St. Pétersbourg T. 11; durch Repertorium der Physik 19, 525. 
und richtet den Apparat nach der Sonne oder dem helleń Himmel. Man erblickt dann direct die Fraunhofer'schen Linien und kann sich leicht orientiren. Man stellt nun das Charnier fest, setzt das Ni col'sche Prisma wieder ein und kann jetzt den Apparat mit jeder gewöhnlichen Lampe beleuchten. Stellt man das Beobachtungsfernrohr auf die Fraunhofer'sche Linie D ein, so erhält man mit dem Apparat genau dieselben Resultate wie mit der früheren Form bei Anwendung von Natriumlicht.

Als eine Flüssigkeit von sehr hohem specifischem Gewicht und grossem Brechungsvermögen ist von $\mathrm{C}$. $\mathrm{Roh} \mathrm{rbach} *$ ) eine concentrirte Lösung von Baryumquecksilberjodid zu den Zwecken empfohlen worden, für welche auch die in dieser Zeitschrift 20,391 und 392; 21, 240; 23, 51 besprochenen in Vorschlag gebracht worden sind. Ich muss mich bei dem wesentlich petrographischen und physikalischen Interesse des Gegenstandes hier mit dem Hinweis auf das Original begnügen.

\section{Das Verhalten des Glycerins zu gewissen ätherischen Lösungen} hat C. Meh ${ }^{* *}$ ) studirt und gefundep, dass das Glycerin eine Anzahl von Körpern, z. B. Eisenchlorid und -bromid, Goldchlorid, Urannitrat, Quecksilberchlorid und Methylviolett besser löst als Aether, so dass man diese Substanzen durch Schütteln der ätherischen Lösung mit Glycerin dem Aether entziehen kann, wenn auch nicht bei allen bis auf die letzten Spuren.

Bei diesen Körpern wird auch durch die Anwesenheit von Glycerin in einer wässrigen Lösung ganz oder theilweise verhindert, dass sie beim Ausschütteln mit Aether in diesen übergehen.

Zur Darstellung reiner Bromwasserstoffsäure in kleinem Maassstabe empfiehlt W. Grüning***) $100 \mathrm{~g}$ gröblich zerriebenes Bromkalium und $280 \mathrm{~g}$ Phosphorsäure von 1,304 specifischem Gewicht in einem etwa $1 / 2 l$ fassenden Glaskolben zu erhitzen. Anfangs löst sich das Salz auf, scheidet sich dann beim Eindampfen wieder theilweise aus und bedingt dann ein (nicht bedenkliches) Stossen beim Sieden, schliesslich löst sich alles wieder zu phosphorsaurem Kali auf und die

*) Annalen der Physik und (hemie [N. F.] 20, 169.

**) Journ. de Pharm, et de Chim. 8, 338; durch Chem. Centralblatt [3. F.] 14, 712 .

***) Pharmaceut. Zeitschr. für Russland 22, 313. 